\title{
A Threat Model for a Cloud Infrastructure with no Hypervisor
}

\author{
William A. R. de Souza, Allan Tomlinson \\ Information Security Group \\ Royal Holloway, University of London \\ Egham Hill, Egham, United Kingdom
}

\begin{abstract}
Cloud architectures capitalise on the many benefits of virtualisation. The central component of virtualisation is the hypervisor, which plays a fundamental role in the virtualised environment. Thus, a hypervisor is typically a complex and large piece of software. The NoHype architecture is a new approach to the security problems related to hypervisors and proposes simply to eliminate the hypervisor. However, as with any new approach to security, it can also introduce new threats. In this paper we conduct an investigation of the NoHype architecture, considering the new data flows, processes, entities, data stores and boundaries introduced by it. We point out that this new architecture does not mitigate all threats that a hypervisor is prone to in cloud architecture, and may even introduce new threats.
\end{abstract}

\section{Introduction}

Cloud computing provides an infrastructure for customers to run their applications and store their information. It allows several virtual machines, from different customers, to exist on the same physical machine capitalising on economy of scale, in a dynamic and scalable computational environment at a cost affordable for customers. Although this is the main appeal of a cloud infrastructure it is also the main concern for customers, since the shared environment is prone to vulnerabilities that may be exploited by a malicious party [2] [3]. Thus, a malicious virtual machine (VM) can start an attack against the whole infrastructure as: a VM running on the same server, the hypervisor or the underlying hardware; potentially exploiting a wide range of vulnerabilities [4].

The hypervisor plays a major role in the virtualised environment and it is a prime target for attackers. The literature presents successful attacks against it, as in the case of a successful execution of code on the host from a guest OS in a VMware environment [5] and an exploitation of the Xen hypervisor that allows the injection of a backdoor facility [6].

There are many approaches to mitigate those threats but they have drawbacks that can hinder their adoption in a cloud environment. For example, one approach is adding extra code to the hypervisor in order to verify its integrity [7], but this enlarges the attack surface and causes overhead to the system [8]. The approach of minimizing the hypervisor [9] [10] diminishes the attack surface, leaving just the essential functionality in the hypervisor. However this can cause lack of functionality in the hypervisor. Hardening the hypervisor is the most common approach [11] [12] [13] and although it sounds obvious it is not always the best solution, since it may enlarge the attack surface, cause overhead to system and, in general, just mitigate specific threats.

The 'no hypervisor' strategy [14] [15] proposes a new approach: rather than defending the hypervisor, just remove the attack surface by getting rid of the hypervisor; but preserving the semantics of virtualisation. In [14] [15], the authors present an architecture called NoHype that is focused on cloud computing. By examining the NoHype threat model in [15], we notice that important new entry points [16] to the cloud infrastructure arise. Moreover, as with any new approach to security, it can introduce new threats to the cloud (or virtualised) environment, which could impede the adoption of the new architecture. Thus, a threat modelling process will help to identify weaknesses and strengths in the architecture, and provide a basis for investigating threats and testing new vulnerabilities [16].

In this context, this work investigates the no hypervisor architecture, identifying entry points which can be attacked directly or used to enable other attacks. Using Data Flow Diagrams (DFD), we review the data flows within the system, the processes transforming these data, the entities interacting with processes, the data stores containing important information for the operation of the system and the types of boundaries in the proposed architecture. We also rate the threats identified in the system expanding the DFD for the high rating threats. We point out that this new architecture does not mitigate all threats that a hypervisor is prone to, and may even introduce new threats.

The remainder of this work is organised as follows. In Section 2 we explain the main entry points in a hypervisor that can enable an attack. In Section 3 we present the NoHype architecture. In section 4 we investigate the no hypervisor architecture pointing out the threats identified and we present a conclusion and future work in Section 5. 


\section{Main entry points in hypervisors}

The hypervisor has elevated privileges in order to perform its functions. Two required features of a hypervisor are security, since it is a main target for attacks, and resource scalability on-the-fly, i.e. the hypervisor should be able to allocate more resources from the host system without stopping the VM that needs the resource. The hypervisor manages all external interactions of VMs, including access to host resources. External interactions are done by means of VM exits. Consequently, the communication among different VMs or VMs and other components in the infrastructure is done indirectly through the hypervisor, by means of VM exits [15].

A VM exit is a trap-and-emulate virtualisation implementation, similar to what happens in operating systems. It occurs when the VM's code tries to execute a privileged instruction. When this happens VM exit occurs, the VM execution is interrupted (trapped) and the hypervisor takes over execution to handle the privileged instruction (emulate) [1]. VM exits are rather frequent. As one can see in [15], in an idle VM running on Xen 4.0, VM exits occur $\sim 600$ times/s.

In this case, an attacker using a malicious VM could force a VM exit to occur, trying to simulate an execution of privileged instructions, and either inject malicious code or cause a malfunction in the hypervisor. By doing this, a successful attacker could violate the confidentiality, integrity and availability of other VMs, of the hypervisor and ultimately compromise the entire cloud infrastructure.

\section{NoHype architecture}

The main idea of the NoHype architecture, as proposed in [14] and [15], is to eliminate the hypervisor attack surface altogether. Thus, getting rid of the hypervisor, one can get rid of the attacks that a hypervisor would be vulnerable to. It means that there are no more VM exits since the virtual machines have no hypervisor to interact with. Nonetheless, it is necessary find other ways to perform the hypervisor functions and to address privileged instructions. The NoHype architecture has four stages: Creation, Bootup, Disengagement and Execution/Shutdown. Those stages occur during the VM's lifetime the system.

It should be noted that the NoHype architecture preserves the semantics of virtualisation, in the sense that it is possible to run and manage virtual machines as is done in the other kinds of cloud infrastructures.

Although today's commodity hardware can be used to host NoHype, this hardware should be modern x86 architecture, since NoHype considers components of the hardware architecture in its operation. But, no special hardware is necessary to do this.

\subsection{NoHype key ideas}

NoHype considers the functionalities of a hypervisor in today's cloud and provides all of them by another means, capitalising on the cloud architecture and on the resources available in modern commodity hardware. In this way it eliminates the attack surface [14] [15]. The following are the key ideas behind NoHype:

\section{- Key idea 1: Pre-allocate memory and cores}

The hypervisor dynamically manages the memory and processor cores, so VMs can be promised more resources than are actually physically available. However in the cloud the customer specifies the resources needed before a VM is created, thus NoHype can pre-allocate processor cores and memory, enforcing memory isolation by means of hardware paging mechanisms, e.g. the extended page table (EPT).

\section{- Key idea 2: Use only virtualised I/O devices} Virtualisation software emulates I/O devices. In NoHype the I/O devices are virtualised, since there are just a few devices needed in the cloud infrastructure, e.g. network connection (NIC), storage, and graphics card (these kind of cards can be used as an extra computing resource). So, it can dedicate each $\mathrm{I} / \mathrm{O}$ device to each VM, eliminating the need for management.

- Key idea 3: Short-circuit the system discovery process

In general, in order to run on different platforms, an OS needs to discover the configuration of the host system. A guest OS running in a VM needs do the same. NoHype uses a temporary hypervisor and a modified guest OS (provided by the cloud infrastructure) to perform hardware discovery during the bootup process, caching the system configuration data for later use.

\section{- Key idea 4: Avoid indirection}

Hypervisors need to map the virtual view to real hardware (indirection). NoHype dedicates processor cores to a VM. So, for instance, a VM can access the real processor ID, eliminating the need for indirection.

\subsection{The Threat Model for NoHype Architecture}

The objective of NoHype is to protect the cloud infrastructure against attacks perpetrated through or against the hypervisor by guest VMs. The threat 
model [16] of the NoHype architecture is shown in figure 1 as a Data Flow Diagram (DFD), since many software attacks involve the data flow through the system [19].

A threat model, as proposed in [16], basically consists of an entity (e.g. "Cloud Provider") that interact with a process (e.g. "1.1 Modify OS for NoHype"), sending data flows (e.g., "Requirements") and, sometimes, storing and querying data from a data store (e.g. "VM Exit Condition", in figure 2). The threat model can also present boundaries (e.g. "Cloud provider boundary (trust)"). Boundaries are important in DFD based threat analysis since this is where data moves between two trust domains. All entities, processes, data flows, data stores and boundaries represented in a threat model bring security concerns.

In our threat model, we can see that the cloud infrastructure provider, the cloud management software and the modified guest OS (key idea 3, above) are assumed not to be malicious and they are included within a trust boundary. The cloud provider modifies the guest OS, according to NoHype's requirements, and makes it available to customers.

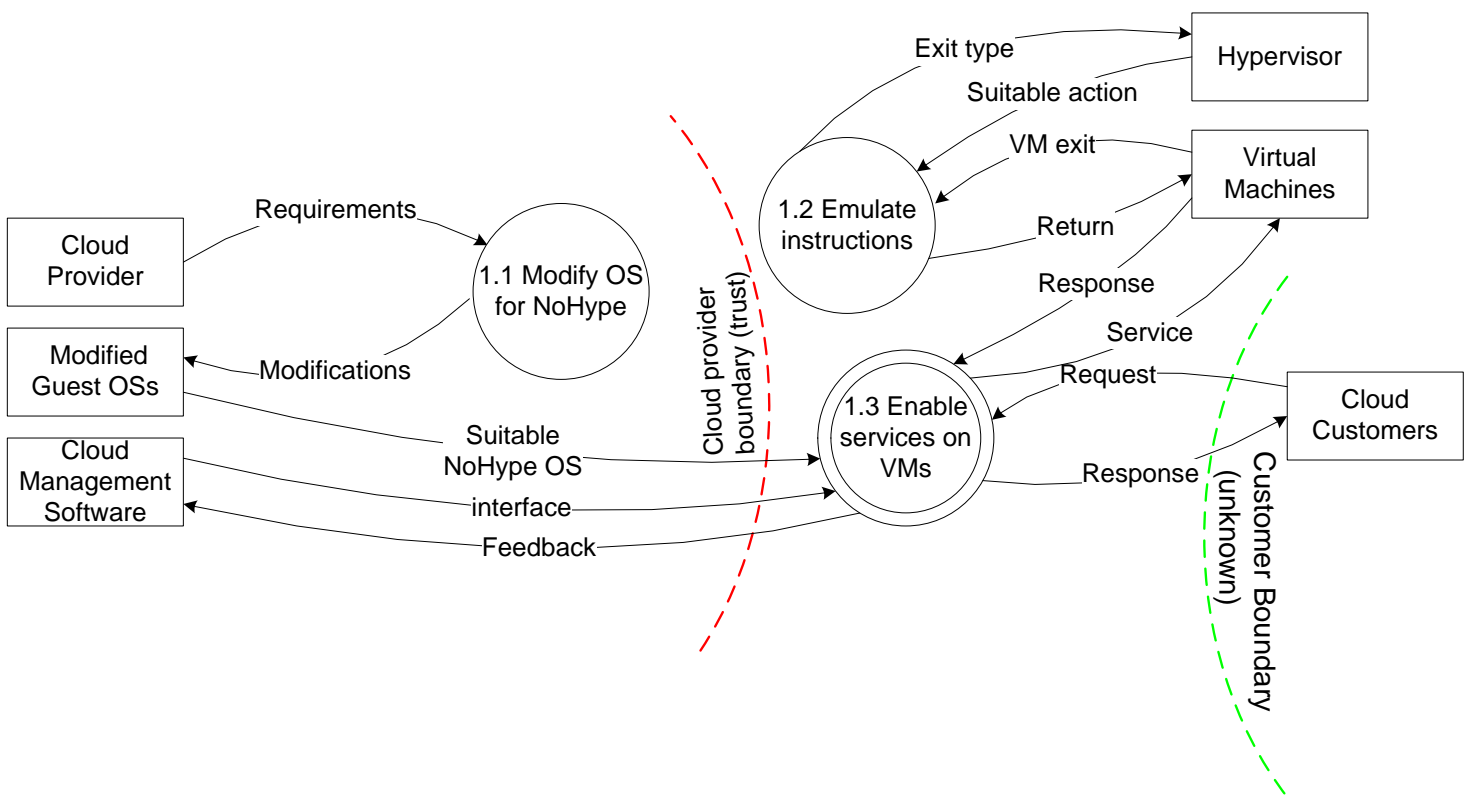

Fig. 1. Threat model for NoHype architecture.

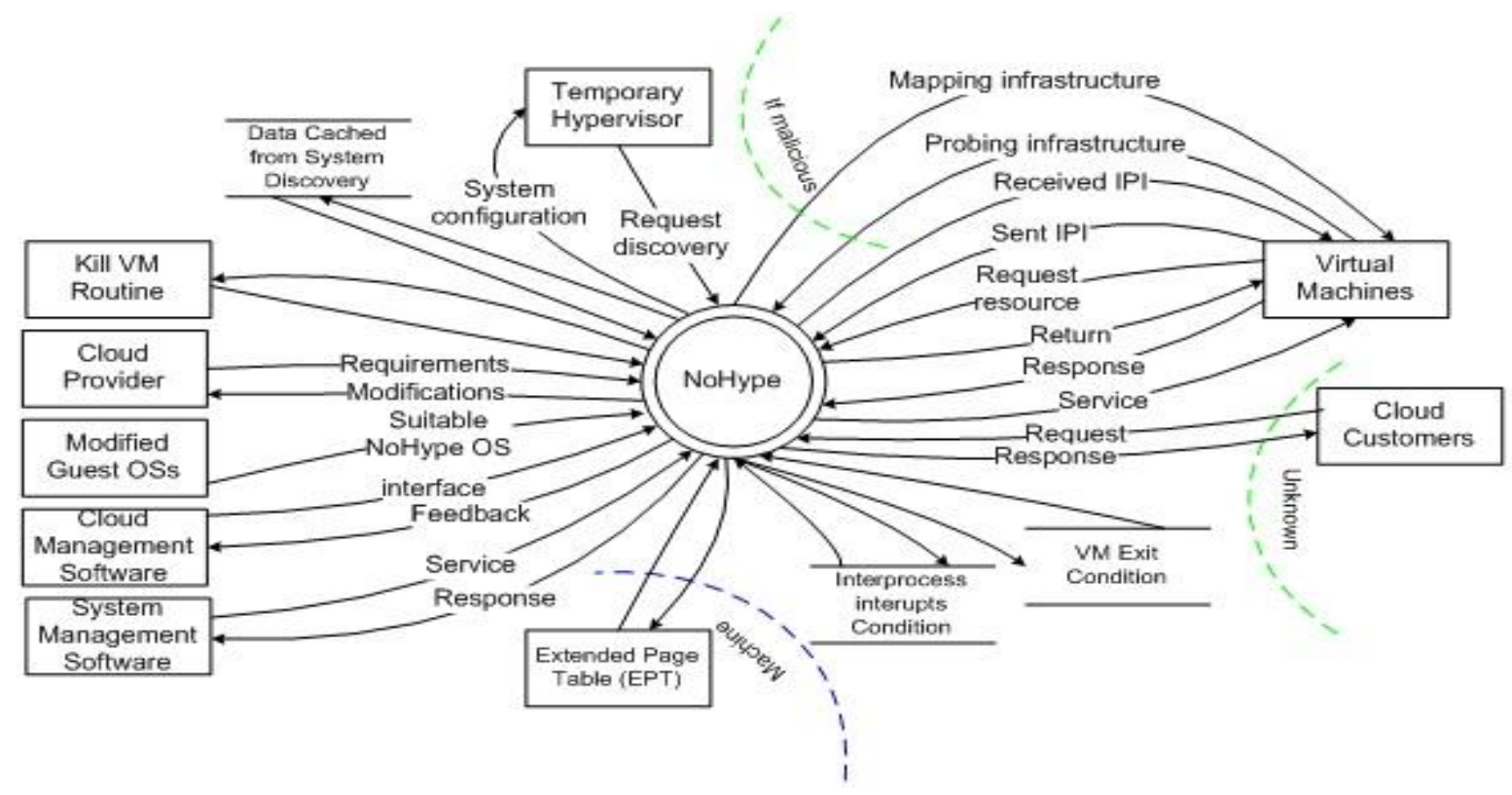

Fig. 2. DFD (context diagram) for a complementary threat model for NoHype. 
The process "Modify OS for No Hype" is responsible for this task. The cloud management software offers an interface for customers to manage their VMs. The multiple process "Enable services on VMs" allows starting, stopping, migrating and all other services related to a VM. A VM starts a VM exit, as described in section 2 , so the hypervisor identifies the exit type (privileged instruction) and executes the appropriate action by means of the process "Emulate instructions". After completing the action, the hypervisor returns the control of execution to the VM.

The threat model makes no assumption about the customer, other than the responsibility for protecting their applications in a VM. So, the customers are included in an unknown boundary.

\section{Investigating the no hypervisor architecture}

Since a threat model can help to investigate threats and vulnerabilities, such that vulnerabilities can be tested and mitigated, we extend the threat model presented in figure 1. Doing so, we expose new data flows, processes, entities, data stores and boundaries introduced by NoHype. It allows better understanding of matters of security in the no hypervisor architecture. The extended threat model is presented in figure 2. It is presented as a DFD context diagram [16], the highest level in a DFD. Of course more detailed analysis could be presented but due to space limitations we focus on the context diagram to illustrate the principles.

The methodology used to identify the new threats is that proposed in [16] [19] and consists of detailed analyses and review of the architecture.

As we can see in figure 2, no trust boundary is set in this new model. In this way, we can do a more complete investigation of security and identify other threats, although we cannot guarantee that we are covering all of them. The multiple process "NoHype" represents all process in the NoHype system.

\subsection{Threats in the architecture}

We describe the threats below, according to the entities, processes, data flows, data stores and boundaries represented in figure 2 , and consider the previous NoHype analysis conducted in [15] and [18].

- Threat 1 - Compromise the System Management Software. An attacker takes over of the "System Management Software"

The entity "System Management Software" is necessary to perform some of the hypervisor tasks, as the VMs cannot execute privileged instructions. It creates a VM after a request of the entity "Cloud Management Software". Thus, it is an import entry point and target for attacks, since it is a kind of minimal hypervisor. The threats to the system management software could come from the interaction with other entities of the cloud infrastructure, such as the "Cloud Management Software", the "Cloud Provider" or the "Modified Guest OS", which could be previously compromised by a malicious party. By acting as the System Management Software an attacker can request the creation of as many malicious "Virtual Machine" as they wish and can execute privileged instructions in order to compromise a target "Virtual Machine".

- Threat 2 - Unauthorized access to resources in the infrastructure. An attacker gains access to the "Cloud Provider", the "Cloud Management Software" or the "Modified Guest OS"

In the proposed model, those entities are no longer included within a trust boundary, since they are considered targets for attacks. An attacker can gain control of one of those entities and use it as vector for attacks or deny its use. For example, an attacker can use: the "Cloud Provider" to create a malicious "Modified Guest OS", the "Cloud Management Software" to create one or more malicious "Virtual Machine", the malicious "Modified Guest OS" to exploit and advance their attack.

- Threat 3 - Disabling or impersonating the Kill VM Routine. An attacker disables or impersonates the "Kill VM Routine"

The entity "Kill VM Routine" is a NoHype piece of code that is triggered any time a VM does some illegal action and consequently causes a VM exit, as implemented in NoHype. So, in the case where an attacker gains control of the "Kill VM Routine", this attacker can impede the routine from acting when a "Virtual Machine" causes a VM Exit, or the attacker could kill any target "Virtual Machine". Examples of illegal actions are a "Virtual Machine" tries executing a privileged instruction or tries to access resources not allocated for it.

- Threat 4 - Modification of the VM Exit Condition. An attacker modifies the flag "VM Exit Condition"

A VM exit is illegal in the system. But, since NoHype needs a "Temporary Hypervisor", a VM exit is not always illegal. As soon as the guest VM is disengaged, a flag ("VM Exit Condition" data store) in the memory is set, indicating the illegal condition of the VM exits. So, an attacker could change this flag to allow VM exits and compromise the system, by allowing a malicious "Virtual Machine" to 
perform any kind of instruction in the system, potently doing what the attacker wants.

- Threat 5 - Compromise the Temporary Hypervisor. An attacker takes over the "Temporary Hypervisor"

In NoHype a "Temporary Hypervisor" is used for the system configuration discovery during the bootup process. Although the VM guest is disengaged from the hypervisor before the VM guest is able to execute its code, if an attacker gains control of the "Temporary Hypervisor" by means of a previous attack performed through one of the entry points described in the previous section, all proposed security for NoHype could be compromised.

- Threat 6 - Tampering with Data Cached from System Discovery. An attacker tamper the "Data Cached from System Discovery" in the memory

In order to avoid the need for a hypervisor during the lifetime of the guest VM, the data collected during the system discovery process is cached ("Data Cached from System Discovery" data store); then a guest VM can query the data as often as it needs. An attacker can modify that data by means of a malicious VM, inserting any suitable information to advance in their attack.

- Threat 7 - Disclosure of infrastructure information. An attacker maps the infrastructure of the cloud

Since NoHype eliminates the hypervisor, VMs are closer to the underlying hardware. Thus, a malicious "Virtual Machine" could map the underlying hardware infrastructure and perform side-channel attacks, as described in [17]. In this way, a malicious "Virtual Machine" could identify where its target "Virtual Machine" is performing in order to attack it. This fact, sets the new boundary "If malicious" in the DFD, indicating that the flows "Probing infrastructure" and "Mapping infrastructure" just occurs when a "Virtual Machine" is malicious.

- Threat 8 - Denial of Service. An attacker modifies the flag "Interprocess Interrupts Condition" and launches a denial of service attack against the "System Management Software" or against a target "Virtual Machine"

In NoHype, a VM can send interprocessor interrupts (IPI) to other cores, as much as it wishes. As a consequence, a malicious VM can send several IPI for a core where a target VM is running or for the core 0, where the "System Management Software" is running, as defined by NoHype architecture. Thus, NoHype uses a flag ("Interprocess Interrupts Condition" data store), for each type of IPI, in a shared region in the memory in a manner that a VM sending an IPI can set the flag and the VM receiving the IPI can check and clear the flag. So, a VM receiving an IPI can ignore this IPI, if the flag is not set. Since the region in memory that holds the flag is shared, an attacker (a malicious VM) can access it and modify it and launch denial of service attacks against their targets. Besides, it is not clear how a "Virtual Machine" can differentiate a legal IPI from an IPI sent by an attacker and since an attacker can be a (malicious) "Virtual Machine", they can set his own flags and the VM receiving the IPI can do nothing about this, but receive the IPI.

- Threat 9 - Unauthorized access to the memory. $A$ malicious "Virtual Machine" accesses the memory region of another "Virtual Machine"

The isolation in the NoHype is dependent on hardware mechanisms. Especially in the case of memory, isolation heavily depends on the correct functioning and implementation of the extended page table (EPT) in order to guarantee confidentiality and integrity. Thus, the EPT is a critical point in the system and beyond the control of NoHype. So, it is included in the "Machine" boundary.

\subsection{Rating the threats with the DREAD model}

Using the Microsoft DREAD model [16], we rating the threats identified in the previous session to prioritise the mitigation tasks according to risk.

The DREAD acronym stands for: Damage potential (how bad is it if the vulnerability is exploited?), Reproducibility (how easy is it to reproduce the attack?), Exploitability (how easy is it to launch an attack?), Affected users (how many users are affected?) and Discoverability (how easy is it to find the vulnerability?).

Following the threat rating table present in [20] we build a DREAD rating table (table 1) for the NoHype system. The total ranges from 5 to 15 . According to [20] we can rate threats as follow: 12 to 15 as High risk, 8 to 11 as Medium risk, and 5 to 7 as Low risk.

By observing table 1, we can notice that threats 3 , 4 and 6 are rated as high; threats 1,2, 5, 7 and 8 are rated as medium; and threat 9 is rated as low. Therfore we will expand the threat model presented in figure 2 to show more details of the components involved this environment.

\subsection{Expanding the threat model for the high rating threats}

The idea behind expanding the threat model is to consider in more detail the data flows, the processes, the entities, the data stores and the types of boundaries that take part in those threats. Thus, figures 3 and 4 shows this expansion. 
TABLE I. DREAD RATING FOR NOHYPE SYSTEM THREATS

\begin{tabular}{|c|c|c|c|c|c|c|c|}
\hline Threat & D & $\mathbf{R}$ & $\mathbf{E}$ & $\mathbf{A}$ & D & Total & Rating \\
\hline Threat 1 - An attacker takes over of the "System Management Software". & 3 & 1 & 1 & 3 & 1 & 9 & Medium \\
\hline $\begin{array}{l}\text { Threat } 2 \text { - An attacker gains access to the "Cloud Provider", the "Cloud } \\
\text { Management Software" or the "Modified Guest OS". }\end{array}$ & 3 & 1 & 1 & 3 & 1 & 9 & Medium \\
\hline Threat 3 - An attacker disables or impersonates the "Kill VM Routine". & 3 & 3 & 2 & 2 & 3 & 13 & High \\
\hline Threat 4 - An attacker modifies the flag "VM Exit Condition". & 3 & 3 & 2 & 2 & 3 & 13 & High \\
\hline Threat 5 - An attacker takes over of the "Temporary Hypervisor". & 3 & 2 & 2 & 2 & 2 & 11 & Medium \\
\hline $\begin{array}{l}\text { Threat } 6 \text { - An attacker tamper the "Data Cached from System Discovery" in the } \\
\text { memory. }\end{array}$ & 2 & 3 & 2 & 2 & 3 & 12 & High \\
\hline Threat 7 - An attacker maps the infrastructure of the cloud. & 1 & 3 & 2 & 3 & 2 & 11 & Medium \\
\hline $\begin{array}{l}\text { Threat } 8 \text { - An attacker modifies the flag "Interprocess Interrupts Condition" and } \\
\text { launches deny of service attack against the "System Management Software" or } \\
\text { against a target "Virtual Machine". }\end{array}$ & 1 & 3 & 2 & 2 & 1 & 9 & Medium \\
\hline $\begin{array}{l}\text { Threat } 9 \text { - A malicious "Virtual Machine" accesses the memory region of } \\
\text { another "Virtual Machine". }\end{array}$ & 2 & 1 & 2 & 2 & 1 & 7 & Low \\
\hline
\end{tabular}

In figure 3, we can observe in detail the interaction between the Virtual Machine and the Kill VM Routine. The Virtual Machine can access all resources allocated for it. But, any time the Virtual Machine produces a VM Exit, the Kill VM Routine is triggered. Then, this routine can query the VM Exit Condition flag to verify if in that moment a VM Exit is illegal or not. If the VM Exit is illegal, the Kill VM Routine shuts down the Virtual Machine that produced the VM Exit. If the VM Exit is legal, the Kill VM Routine does nothing.

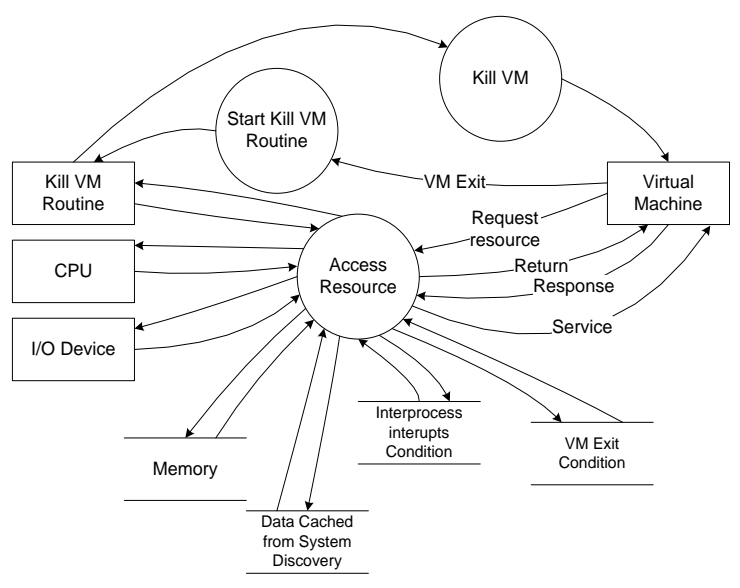

Fig. 3. DFD (Level 0) for threat 3

In figure 4 , we can observe the DFD for threats 4 and 6. After the discovery process, the Temporary Hypervisor stores the result of the search in a cache. After concluding its task a Guest OS Kernel Module sends commands to disengage the Temporary Hypervisor. The Virtual Machine receives a message about the disengagement of the Temporary Hypervisor. After that, the VM Exit Condition flag is set to inform that from this point on any VM Exit will be considered illegal.

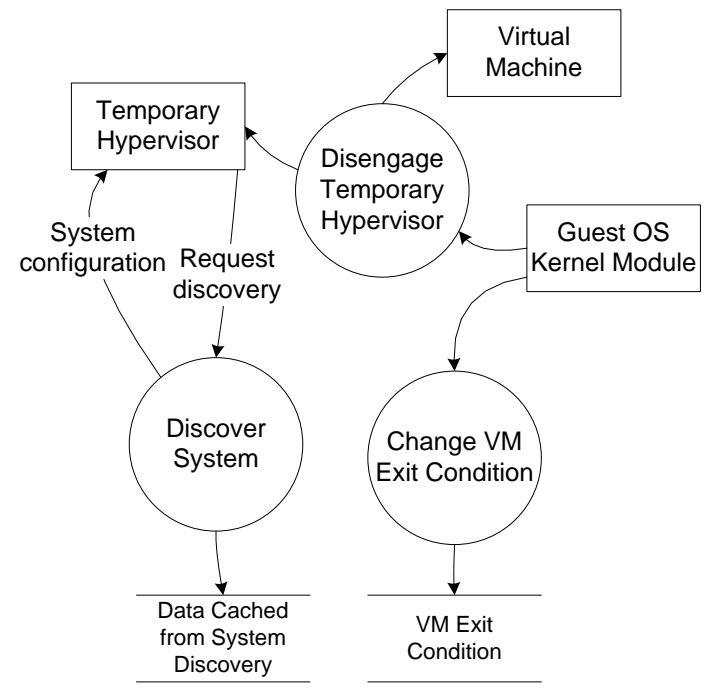

Fig. 4. DFD (Level 0) for threats 4 and 6

\section{Conclusion and future work}

The NoHype architecture proposes a radical new approach to address the matter of security involving the hypervisor: get rid of it! Basically, NoHype identifies the main roles of a hypervisor and searches for some other manner to do the same thing, in order to eliminate the hypervisor.

NoHype is a feasible architecture that can be implemented with today's commodity hardware. But, this new architecture does not mitigate all threats that 
a hypervisor is prone to in cloud architectures and introduces some new threats. All of this is likely to hinder its use in a real world scenario.

Our future work is to investigate how these threats might be mitigated by establishing Data Flow Diagrams in deeper levels for the NoHype architecture, keeping in mind that improvement must be at least as secure as that one proposed in NoHype system.

\section{References}

[1] A. Silberschatz, P. Galvin, and G. Gagne, Operating System Concepts, 9th ed. Hoboken, NJ: Wiley, 2013.

[2] M. Christodorescu, R. Sailer, D.L. Schales, D. Sgandurra, and D. Zamboni, D, "Cloud security is not (just) virtualization security: a short paper," Proceedings of the 2009 ACM workshop on Cloud computing security, ACM, 2009, 97-102.

[3] A.S. Ibrahim, J.H. Harris, and J. Grundy, "Emerging Security Challenges of Cloud Virtual Infrastructure," in Proceedings of APSEC 2010, Cloud Workshop, Sidney, Australia, 20 nov2010.

[4] D. Shackleford, Virtualization Security: Protecting Virtualized Environments. Indianapolis, IN: Sybex, 2013.

[5] K. Kortchinsky, "Hacking 3D (and Breaking out of VMWare)," BlackHat USA, 2009.

[6] R. Wojtczuk, "Subverting the Xen hypervisor," BlackHat USA, 2008.

[7] A.M, Azab, P. Ning, E.C. Sezer, and X. Zhang, "HIMA: A Hypervisor-Based Integrity Measurement Agent," Proceedings of the 2009 Annual Computer Security Applications Conference, IEEE Computer Society, 2009, 461-470.

[8] A. M. Azab, P. Ning, Z. Wang, X. Jiang, X. Zhang, and N. C. Skalsky, "HyperSentry: Enabling stealthy in-context measurement of hypervisor integrity," in ACM Conference on Computer and Communications Security (CCS), pages 38-49, October 2010

[9] A. Seshadri, M. Luk, N. Qu, and A. Perrig, "SecVisor: A tiny hypervisor to provide lifetime kernel code integrity for commodity OSes," SIGOPS Oper. Syst. Rev., 41(6):335350, December 2007

[10] J. M. McCune, Y. Li, N. Qu, Z. Zhou, A. Datta, V. Gligor, and A. Perrig, "TrustVisor: Efficient TCB reduction and attestation," in IEEE Symposium on Security and Privacy, pages 143-158, May 2010.

[11] C. Li, A. Raghunathan, and N. K. Jha, "Secure virtual machine execution under an untrusted management OS," in Proceedings of the Conference on Cloud Computing (CLOUD), July 2010.

[12] R. Sailer, E. Valdez, T. Jaeger, R. Perez, L. V. Doorn, J. L. Griffin and G. S. Berger, "sHype: Secure hypervisor approach to trusted virtualized systems," Technical Report RC23511, IBM Research, 2005.

[13] U. Steinberg and B. Kauer, "NOVA: A microhypervisorbased secure virtualization architecture," in European Conference on Computer Systems, April 2010.

[14] E. Keller, J. Szefer, J. Rexford, and R. B. Lee, "NoHype: Virtualized cloud infrastructure without the virtualization," in International Symposium on Computer Architecture (ISCA), June 2010.

[15] J. Szefer, E. Keller, R.B. Lee, and J. Rexford, "Eliminating the hypervisor attack surface for a more secure cloud," in Proceedings of the 18th ACM conference on Computer and communications security, ACM, 2011, 401-412.

[16] F. Swiderski and W. Snyder, Threat Modeling. Redmond, WA: Microsoft Press, 2004.
[17] T. Ristenpart, E. Tromer, H. Shacham, and S. Savage, "Hey, you, get off of my cloud: Exploring information leakage in third-party compute clouds," in ACM Conference on Computer and Communications Security (CCS), November 2009.

[18] W. de Souza \& A. Tomlinson. Virtualisation without a hypervisor in cloud infrastructures: an initial analysis. in PGNet 2013, The 14th Annual Postgraduate Symposium on the Convergence of Telecommunications, Networking \& Broadcasting, Jun 2013.

[19] A. Shostack. Experiences Threat Modeling at Microsoft. Microsoft, Security Development Lifecycle Blog, 2008. http://blogs.msdn.com/b/sdl/archive/2008/10/08/experiences -threat-modeling-at-microsoft.aspx. Accessed in 22/04/2013.

[20] J.D. Meier, A. Mackman, M. Dunner, S. Vasireddy, R. Escamilla and A. Murukan. (2003) 'Improving Web Application Security: Threats and Countermeasures', Microsoft Corporation; http://msdn.microsoft.com/enus/library/ff648644.aspx (16 January 2014). 